\title{
Service der Verlagsredaktion
}

\section{Der Kanal Business-IT im Wissensportal Springer für Professionals bietet aktuelle Themen sowie fun- diertes Hintergrundwissen für IT-Manager. In der Datenbank finden Sie derzeit rund 40000 Fachbü- cher und 300 Fachzeitschriften aus den Bereichen Wirtschaft und Technik.}

\section{In eigener Sache: Nutzer geben gute Noten für Springer für Professionals}

Dem Wissensportal Springer für Professionals werden ein hoher Praxisbezug sowie authentische und glaubwürdige Inhalte attestiert. Dies ergab eine aktuelle Nutzerbefragung. An der Onsite-Befragung, die zwischen März und Mai dieses Jahres vom Institut eResult durchgeführt wurde, nahmen von März bis Mai insgesamt 1620 Nutzer des Portals teil.

Insgesamt zeichnen sie sich durch einen hohen Bildungsgrad aus und verantworten mehrheitlich leitende Positionen. Die große Mehrheit ist aktiv an den Investitionsentscheidungen in ihrem Unternehmen beteiligt. Inhaltlich schätzen die Nutzer die Themenvielfalt des Portals, die Aktualität sowie die Verfügbarkeit wissenschaftlich und fachlich fundierter Informationen. 75 Prozent der Nutzer haben großes Interesse an Fallbeispielen (Best Practices) sowie Brancheninformationen und -entwicklungen.

Über 70 Prozent der befragten Entscheider geben an, dass die Inhalte des Portals für ihre geschäftlichen Entscheidungen relevant seien. Der Newsletter im Bereich Business IT wird inzwischen von rund 2000 Nutzern abonniert - mit steigender Tendenz. Insgesamt erreicht das Portal Springer für Professionals derzeit durchschnittlich eine Million Page Impressions pro Monat.

\section{Das Wissensportal Springer für Professionals}

Alle Beiträge und Literaturtipps im Heft, die mit Sisp gekennzeichnet sind, sind für Abonnenten des Portals Springer für Professionals im Volltext unter www.springerprofessional.de frei zugänglich. Abonnenten dieser Zeitschrift können das Portal drei Monate kostenfrei unter Angabe des Aktionscodes C0006818 testen und danach zum Vorzugspreis beziehen.

SfP www.springerprofessional.de/fachzeitschriften/
Weiterführende Inhalte aus dem Portal

Aktuelle Studie: Cyber-Kriminalitåt nimmt zu

Trotz steigender Investitionen in die ITSicherheit nimmt die Bedrohung durch Angriffe aus dem Cyberspace weiter zu. Während sich Kriminelle neuester Technologien bedienen, unterschätzen viele Unternehmen die Risiken.

www.springerprofessional.de/4717598

\section{Wie die Einführung von mo-} bile CRM gelingt

Mobile CRM sollte mit einer klaren Strategie im Vertrieb eingeführt werden. Denn vom richtigen Mobility-Konzept hängt der Erfolg im Kundenmanagement ab. Tipps für Ihr Mobile-CRM-Projekt. www.springerprofessional.de/4877804

\section{Digitale Forensik - Spurensi-} cherung im 21. Jahrhundert

Forensik kennt man aus dem Fernsehen. Dass in Zeiten moderner Kommunikationstechnik die Spurensicherung auf technischen Geräten an Bedeutung gewinnt, ist weniger bekannt. Marko Rogge verdient sein Geld damit, herauszufinden, ob etwa mit Smartphones illegale Geschäfte organisiert wurden. www.springerprofessional.de/4691166 